DE DE GRUYTER

OPEN

G

BULGARIAN ACADEMY OF SCIENCES

CYBERNETICS AND INFORMATION TECHNOLOGIES • Volume 14, No 2

Sofia • 2014

Print ISSN: 1311-9702; Online ISSN: 1314-4081

DOI: $10.2478 /$ cait-2014-0020

\title{
Cross Layer Based Congestion Control in Wireless Mesh Networks
}

\section{Ch. Pradeep Reddy, P. Venkata Krishna}

School of Computing Science and Engineering VIT University, Vellore, Tamil Nadu, India Emails: pradeep@vit.ac.in pvenkatakrishna@vit.ac.in

\begin{abstract}
A major issue that attracts the researchers in wireless mesh networks is the issue of performance of the TCP. The role of the congestion control techniques is pivotal in the development of optimal solutions. In wireless mesh networks, the efficiency of bandwidth management in the process of developing optimal solutions depends largely on the congestion control methods used. This paper explores the possibility of such mechanisms which can achieve efficient congestion control in wireless mesh networks. The method proposed is known as Cross Layer Congestion Control (CLCC) and it is developed on the basis of the concept of cross layer optimization. The main purpose of the paper is to provide an efficient bandwidth management scheme for high speed video applications through a congestion control mechanism. The performance evaluation of the proposed method of CLCC for different environments of traffic models, such as single source to single destination and multiple sources to multiple destinations, are appropriate when compared to the existing models.
\end{abstract}

Keywords: Bandwidth management, cross layer, WMN, congestion control.

\section{Introduction}

Wireless Mesh Networks (WMNs) play a pivotal part in the process of evolution of the next generation networks. WMNs can widely be applied in several networking applications, such as high speed Internet applications, video applications, public safety, transport systems, campus or community networks and so on, because of their features of lower cost and faster configuration. All these networks encounter the major problem of managing the bandwidth. In WMN [1-3] we have mesh clients and a wireless backbone, consisting of mesh routers and gateway nodes, 
which form the back-haul links for providing Internet connectivity. The status of mesh clients could either be mobile or stationary. The backbone can provide a service to a variety of networks, such as Mobile Ad-hoc NETworks (MANETs), Ad-hoc networks, WLANs, cellular networks, wireless sensor networks, etc. WMN established its credentials in providing the last mile access to different services. There is a possibility for the mesh routers to be mobile. But we focus only on the infrastructure/backbone mesh network, a very largely used network structure, such as in community networks [1].

The manageability of different applications [4-14] makes the provision of good bandwidth support for wireless mesh networks, a very challenging task. Several wireless nodes in the vicinity of each other share the bandwidth. While some aggressive nodes dominate the channels, some others may fail to get sufficient bandwidth for the requirement of their services. Hence, by constituting proper regulatory mechanisms, the bandwidth must be scrupulously managed. The primary issues in bandwidth management $[7,8]$ include efficient bandwidth utilization through proper allocation of channels, reduction at the levels of congestion, estimation and evaluation of the bandwidth available at any point of time, wireless channel monitoring, adoption of the flow and rate, and provision of QoS guaranty $[14,15]$. But there is a significant difference between the wired and wireless networks in terms of bandwidth, delay in propagation and reliability of the link. The consequent implication of this difference is that the losses of packets are no longer due to the network congestion only. There could also be some other wirelessspecific reasons.

This mobility in WMNs results in link failures of frequently and consequent packet losses. But TCP traces congestion as the cause for these packet losses. Frequent retransmission timeouts in TCP and its performance degradation even at light loads can be attributed to this incorrect inference. The performance of TCP in wireless Ad hoc networks has been demonstrated as poor in several research works [5-6]. A huge magnitude of packet losses and consequent increased incidents of TCP timeouts of retransmission are the reasons for this degradation in performance. First, a node drops a packet when the packet cannot be forwarded to the next hop in the packet relay route, as the next hop node has moved out of the range of transmission. Congestion in the shared medium can be the second valid reason for packet loss. The degradation in TCP performance could be due to congestion in wire-line networks and mobility in Ad hoc networks even at the instances of light loads [16-39].

The extensive researches on Ad hoc and cellular networks emphasize the fact that WMN is an ideal and largely utilized technology addressing possible future service requirements and applications. This relatively untrodden area is in need of huge research and exploration to identify the various demands and challenges and address them. The past studies have thoroughly explored and analyzed several features of WMNs, such as routing, resource allocation, multicasting, control of congestion, etc [2-7]. Identifying sources that violate conversation and fixing them properly is the major function to be considered in congestion control. Loss of packets can be detected in two ways, either through a retransmission timeout or 
when a marginal volume of duplicate acknowledgement is received. A duplicate acknowledgement is a sure indication of out-of-order packet reception by the receiver, meaning a packet drop [25-35].

In trail drop approach, the packets are dropped by the router when the buffer is found to be full. Two noticeable setbacks in this approach are the global synchronization on one side, and the lockout on the other. Various studies have focused on the issues pertaining to optimization of the mechanisms of congestion control as TCP's default techniques. This is necessary since faster transmission and recovery, which reduce the effect of losses on throughput, are incapable of handling applications sensitive to delay.

Floyd and Van Ja c obs on [29] present Random Early Detection (RED) gateway mechanisms to avoid congestion in packet-switched network. These RED gateways maintain the average queue size low, but allow packet bursts occasionally in the queue. Explicit Congestion Notification (ECN) [29] is based on the capacity of the network to trace out the accumulation of congestion. Contrary to the conventional approaches to avoid congestion based on packet dropping, implementation with ECN in the networks react well during incipient stages of congestion. In congestion elimination schemes like Explicit Congestion Notification [29], packet marking is preferred instead of packet dropping during the early stages of congestion. The senders are informed that receivers accept such marked packets, as a result of which the senders limit and reduce their rate of transmission in accordance with this received information. Depending on the average queue length, the routers mark the packets with probability to avoid excessive congestion.

W e i et al. [2] propose SRAM framework, which is a light weight scheme for the management of bandwidth, while $\mathrm{D} \mathrm{e-N} \mathrm{i} \mathrm{an} \mathrm{Yang} \mathrm{et} \mathrm{al.} \mathrm{[3]} \mathrm{discuss} \mathrm{different}$ methods of policies in efficient allocation of the resources, based on the multicasting techniques for WMNs. K. Nahrstedt et al. [4] illustrate a framework, utilizing the cross layer methods of design for issues related to bandwidth management. M. Iqbal et al. [7] illustrate various issues connected with QoS emerging in the development process of an efficient bandwidth management scheme for WMNS.

P. Venkata Krishna et al. [9, 18] propose CLIASM (Cross Layer Interaction and Service Mapping), which is a QoS model depending on a cross layer particularly for MANETs. Information sharing is proposed in this model in a forward and backward two-way flow mechanism. Even when individual layers need to perform diverse functions, sharing of information becomes possible only through a shared database. By creating twin interfaces between the two layers, this two-way information flow is enabled.

C i c c o n e t $\mathrm{i}$ et al. [36] propose an algorithm called FEBA (Fair End-to-end Bandwidth Allocation), a distributed algorithm for the purpose of balancing bandwidth. This specifically customized FEBA is especially addressed to resolve the issues of the traffic flow, such as the problem of unfairness with varying path lengths, which may adversely affect WMNs. Admission control process in wireless mesh networks [35-39] plays a prominent part in providing assurance of Quality of Service. Estimation of the available bandwidth is difficult due to the existence of a 
hidden terminal. Extending the derivation of the channel business ratio and for the estimation of the bandwidth availability, a novel approach has been developed, which considers the possible impact of the hidden terminals on the multi-hop WMNs [7]. The available bandwidth is first estimated on the basis of the information about the channel and the hidden terminal in order to operate medium access control. Then an Admission Control Algorithm (ACA) is designed in order to address the issues of QoS for the traffic in both real-time and non real-time. All the nodes on a route make an admission control decision on the basis of the estimated available bandwidth for the real-time traffic. An algorithm of the rate adoption is proposed to adjust the rate of transmission of sources for the non realtime traffic, to protect the network against entering a state of congestion [7]. Dyer and Boppana report simulation results of TCP Reno performance (see [7]) over three diverse routing protocols (AODV [3], DSR [4], ADV [6]). ADV is found to be performing well under different patterns of mobility and topologies. Besides, for performance enhancement of the on-demand routing protocols (AODV and DSR), they propose a heuristic technique known as fixed RTO. A study of the performance of TCP Tahoe, Reno, New Reno, Sack and Vegas4 over the multi-hop chain topology in instances using IEEE 802.11 protocol, is taken up by $\mathrm{X} \mathrm{u}$ and $\mathrm{S}$ a adawi [6]. The studies illustrate that TCP Vegas performs better and overrides the deficiency of instability. The method of channel bandwidth sharing in Ad hoc networks is studied in [7] and [8]. The authors report an unfair sharing of bandwidth, using the actual MAC 802.11 in the ambience of multi-hop communication. The cause for this is the fact that congestion does not take place in a single node but affects the entire network area involving several nodes. Hence, the local packet queue is not a valid reflection of the state of congestion of the network. Exclusively for this reason, a novel distribution queue is defined, which contains all such packets, the transmission of which is likely to affect node transmission besides its own packets.

Hence, it motivated us to develop innovative and efficient strategies to control congestion, which can well handle the issues of bandwidth management for WMNs. The basis of our approach is cross layer optimization. The existing references demonstrate the development of numerous approaches on the basis of the cross layer optimization to address the issues relating to routing, channel allocation and assignment in wireless networks.

To address the bandwidth management issue, we propose a cross layer optimization technique for congestion window management of mesh networks dependent on the dynamic incidents of traffic conditions. The performance of our approach has been estimated in relation to the end-to-end delay and improvement in packets delivery and the same has been compared with Wireless Control Protocol with Capacity estimation (WCPCap) [5]. WCPCap is specifically selected since it is developed for various mesh topologies, where the main concept is the use of rate control methods with near-optimal max-min strategy.

The following are some of the major contributions of the paper:

- Development of an efficient congestion control method for WMN. 
- Cross Layer based Congestion Control (CLCC) mechanism, providing higher bandwidth.

- Estimation of congestion for different traffic models as single source to single destination and multiple sources to multiple destinations.

- Performance analysis of CLCC.

The organization of the rest of the paper is as detailed:

Section II explains the network design and the system design of the proposed framework with cross layer design. Section III illustrates with a sample example how the current model performs for various traffic scenarios. Section IV presents a comparative analysis of our approach with the existing approaches. Section V concludes the efficiency of the proposed work and the probable future works are explored.

\section{System model}

Several networks are inter-linked based on mesh topology. The routers of one network, transmitting packets are mutually interconnected with the other routers in different networks in the form of a mesh network. Fig. 1 illustrates the topology under consideration for evaluation of the proposed algorithm. In this current model four interconnected networks are considered through four Boundary Routers (BR), distinctly identified as BR1, BR2, BR3 and BR4. Only the boundary routers from a particular node transmit the packets to another specific node in a different network. The proposed algorithm and the mesh network in the threshold routers are employed for evaluation of the CLCC.

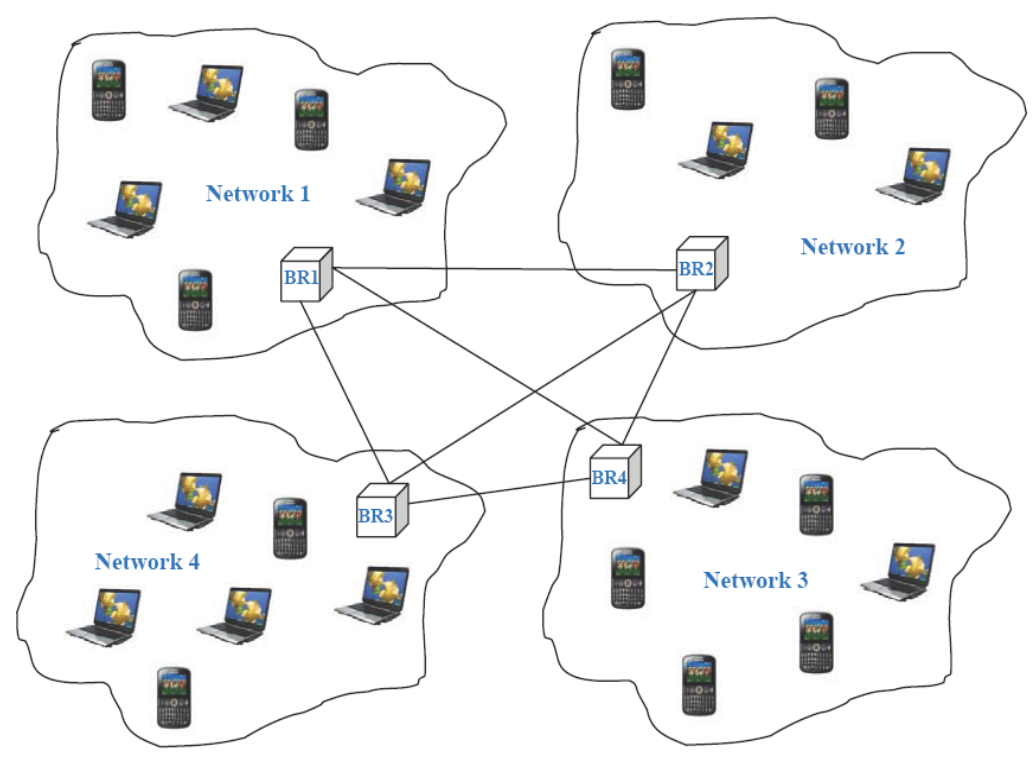

Fig. 1. Network topology 
Two different types of traffic, real-time and non real-time are considered here. Two distinct cases of data transfer, single source to single destination and multiple sources to multiple destinations are taken into consideration.

Cross layer architecture. We propose a cross layer architecture involving the MAC layer and Transport layer in this paper. The level of congestion is the communicated parameter in these two layers as illustrated in Fig. 2.

The level of congestion of every possible path is considered and estimated in the MAC layer and then it is communicated to the transport layer utilizing the proposed cross layer architecture, where the control of the call admission initiates necessary measures to admit the calls on the basis of the traffic type - non real-time or real-time traffic. Such path, where the congestion is absent or minimal is chosen for admission of the real-time traffic and bandwidth to the fullest, allocated to it, for the real-time traffic is treated as high priority flows. In the same way, the path with moderate estimated congestion levels is utilized to admit non-real time traffic also when the levels of congestion become high. The whole process is repeated, but with a decreased size of the congestion window. Every time the process is repeated, the size of the congestion window is reduced by $25 \%$. At the initial stages we maintain the same congestion window size constantly for every flow. The reduction in the congestion window size occurs only in case the estimated congestion levels are high in the chosen path and the process requires repetitions. This cross layer technique is utilized for optimization of the congestion control mechanism.

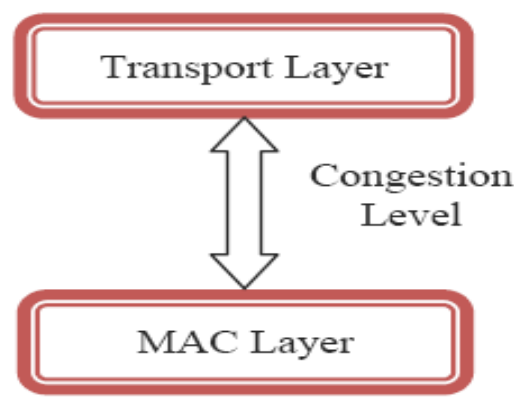

Fig. 2. Cross layer architecture

Finally, when the path is chosen for transmission of data, the size of the congestion window of each node along the chosen path is maintained to be unique. In order to avoid packet drops, a small size queue needs to be maintained. In case the size of the congestion window of all the nodes is not maintained as unique, there is a possibility of occurrence of congestion, since the bandwidth of the distinct edges might be different from one another. In case the bandwidth of the before edge is more, the rate of sending this node is assumed to be high and an increased number of packets will arrive at a node having a low rate of sending and the possibility of congestion occurrence enhances at this stage. Hence, a unique congestion window size is maintained, so that the rate of sending all nodes would be the same. 


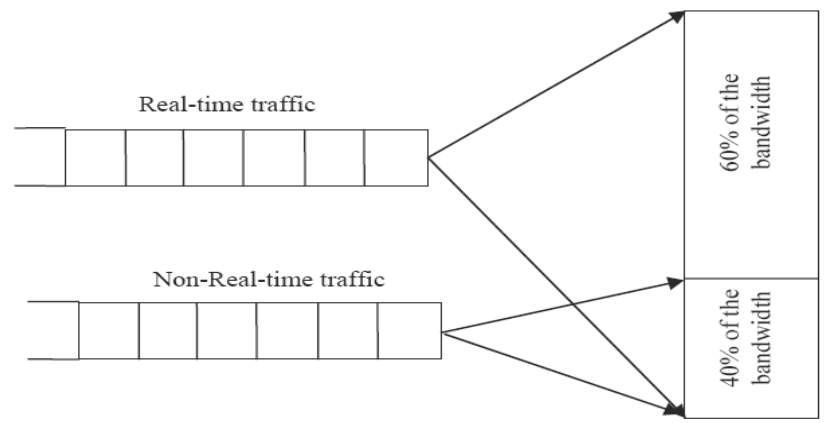

Fig. 3. System model

Fig. 3 shows the system model, demonstrating the usage of the bandwidth available for various types of calls. The real-time traffic gets a reservation of $60 \%$ of the available bandwidth, which cannot be utilized for the non real-time traffic transmission. This indicates that only limited bandwidth is permitted for the utilization of the non real-time traffic. Hence, larger queues are maintained in order to reduce or avoid possible congestion in case of non real-time traffic. If the total permissible $40 \%$ of the available bandwidth is utilized in case of the non real-time traffic, then there is a possibility of occurrence of congestion.

\title{
3. Cross layer based congestion control
}

Algorithms used in controlling congestion in wireless mesh networks are presented in this section. There are two possible variations of the CLCC on the basis of the type of source of traffic, such as a single source and single destination and multiple sources and multiple destinations.

\author{
Algorithm 1. Single source and single destination \\ Input \\ Source node $-\mathrm{s}$ \\ Destination node $-\mathrm{d}$ \\ Required bandwidth $-\mathrm{B}_{\mathrm{R}}$ \\ Begin
}

Step 1. Determine all the possible paths between s and $d$

Step 2. Determine the available bandwidth, $B_{A}$ for each path determined in Step 1

Step 3. Congestion level of each path calculated as $\left(B_{R}-B_{A}\right) / B_{R}$

Step 4. If congestion level $<=0$ then

\section{Step 5. Else}

4.1.1. Indicate there is no congestion along the particular path

\subsubsection{Congestion level is estimated}

Step 6. The path with less congestion is chosen as an optimal path

End

Output

Optimal path between the source and destination 
In case of single source and single destination, the transmission can be done by any source in the network to any of the nodes in the network, which becomes a destination node. The type of data to be transmitted can either be real-time or non real-time traffic. The available bandwidth for each path is determined for all the possible paths from the particular source node to a specific destination node, in the process of either real-time or non real-time flow from the source node to the destination node for a successful transmission of the flow. The levels of congestion for all possible paths are estimated based on the information determined.

\section{Algorithm 2. Multiple sources and multiple destinations \\ Input}

Source nodes $-\mathrm{s}_{1}, \mathrm{~s}_{2}, \ldots, \mathrm{s}_{n}$

Destination node $-\mathrm{d}_{1}, \mathrm{~d}_{2}, \ldots, \mathrm{d}_{n}$

Required bandwidth $-\mathrm{B}_{\mathrm{R} 1}, \mathrm{~B}_{\mathrm{R} 2}, \ldots, \mathrm{B}_{\mathrm{R} n}$

Begin

Step 1. Determine all the possible paths between $s_{i}$ and $d_{i}$

Step 2. Node disjoint test is performed

Step 3. If node disjoint test fails then

3.1.1. Edge disjoint test is performed

3.1.1.1. If edge disjoint test fails then

3.1.1.1.1. Determine the time share among the common links

Step 4. Determine the available bandwidth, $\mathrm{B}_{\mathrm{A} i}$ for each path determined in Step 3

Step 5. Congestion level of each calculated as $\left(\mathrm{B}_{\mathrm{R} i}-\mathrm{B}_{\mathrm{Ai}}\right) / \mathrm{B}_{\mathrm{R} i}$

Step 6. If congestion level $<=0$ then

6.1.1. Indicate there is no congestion along the particular path

Step 7. Else

7.1.1. Congestion level is estimated

End

Step 8. The path with less congestion is chosen as an optimal path

\section{Output}

Optimal Path between the source and destination

Algorithm 2 explains the transmission of either real-time or non real-time traffic from different sources to different destinations. Concepts, such as node disjoint paths and edge disjoint paths are utilized here in order to minimize congestion and enhance the throughput of the system. In case of multiple flows from various sources to be transmitted to different destinations, the bandwidth requirements for different flows and all the possible paths of all the present flows are determined. Then, the test for node disjoint is carried out. Only such possible paths are selected, which satisfy the node disjoint condition. Else, possible paths satisfying the edge disjoint condition are chosen. In the event of both the node disjoint and edge disjoint condition not being satisfied, the determination of sharing of the time of flows along the common links is done on the basis of the rate of arrival of the flows at this common link. The bandwidth available for the selected 
path is then determined and finally an estimation of the level of congestion of the determined path is made.

Estimation of the congestion level. Congestion does not occur when the bandwidth available for a particular path is sufficiently larger than the bandwidth required for the flow. Or, if $90 \%$ of the required bandwidth for the flow is available, the congestion level is considered to be low. If the available bandwidth is inbetween $75 \%$ and $90 \%$, the level of congestion is estimated to be moderate. If the available bandwidth is less than $75 \%$ of the requirement for the flow, then the level of congestion is high.

Illustrative Example. If we consider the mesh network as indicated in Fig. 3 , there are 8 nodes with node 2 acting as a source node and node 8 as a destination node. Each edge is denoted by a value, representing the respective bandwidth of the edge in Mbps.

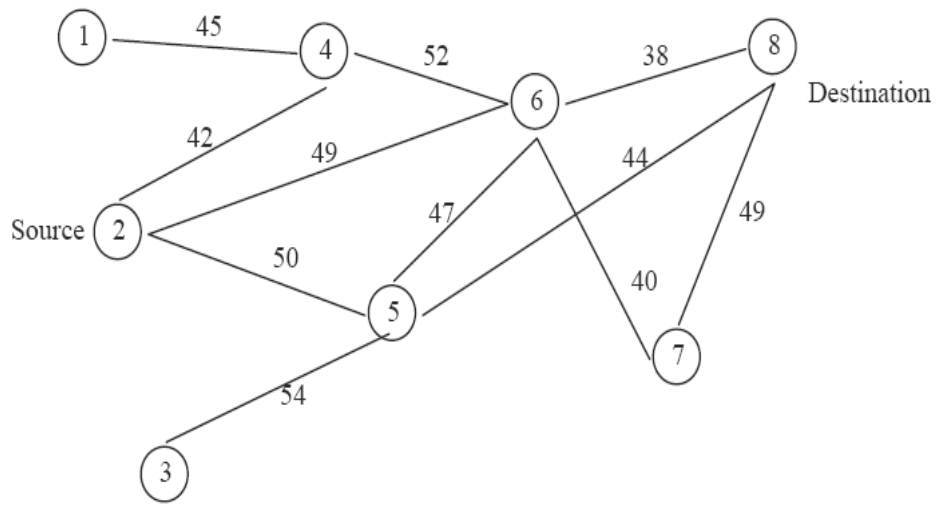

Fig. 4. Mesh network

If we consider the mesh network as indicated in Fig. 3 , there are 8 nodes with node 2 acting as a source node and node 8 as a destination node. Each edge is denoted by a value, representing the respective bandwidth of the edge in Mbps.

Case 1. In single source and single destination: consider that the real time flow requires 42 Mbps bandwidth, the source is node 2 and the destination is node 8 . Then the possible paths from node 2 to node 8 are:

- Path $1_{\mathrm{a}}$ : node $2 \rightarrow$ node $4 \rightarrow$ node $6 \rightarrow$ node 8 (38)

- Path $2_{\mathrm{a}}$ : node $2 \rightarrow$ node $6 \rightarrow$ node 8 (38)

- Path 3 a: node $2 \rightarrow$ node $5 \rightarrow$ node $6 \rightarrow$ node 8 (38)

- Path 4 a: node $2 \rightarrow$ node $5 \rightarrow$ node 8 (44)

- Path $5_{\text {a }}$ : node $2 \rightarrow$ node $5 \rightarrow$ node $6 \rightarrow$ node $7 \rightarrow$ node 8 (40)

- Path 6 a: node $2 \rightarrow$ node $6 \rightarrow$ node $7 \rightarrow$ node 8 (40)

- Path 7 a: node $2 \rightarrow$ node $4 \rightarrow$ node $6 \rightarrow$ node $7 \rightarrow$ node 8 (40)

- Path 8 a: node $2 \rightarrow$ node $6 \rightarrow$ node $7 \rightarrow$ node 8 (40)

- Path $9_{\mathrm{a}}$ : node $2 \rightarrow$ node $6 \rightarrow$ node $5 \rightarrow$ node 8 (44) 
The bandwidth available for the path is provided at the end of the path. For instance, the bandwidth available for path 1 is $38 \mathrm{Mbps}$. At this juncture the level of congestion of all determined paths is estimated. For instance, the congestion level of path $1,2,3$, is 0.0952 and that of paths $5,6,7,8$, is 0.0476 and the congestion level of path 4 and 9 is 0 .

In case of paths 4 and 9, the congestion level is 0 and certainly a larger bandwidth is available here than the actual requirement. Utilizing the cross layer technique, these levels of congestion are communicated and eventually paths 4 and 9 are selected for transmission, since there is minimal or no congestion.

When we consider the second case of multiple sources to multiple destinations, two different transmissions are assumed. The first one is from node 2 to node 8 and the other one - from node 1 to node 8 .

The different paths from node 2 to node 8 are represented as path $n_{a}$, where $n$ is the path number and are given above. The possible paths from node 1 to node 8 are represented as path $m_{b}$, where $m$ is path number and are given below:

Path $1_{b}$ : node $1 \rightarrow$ node $4 \rightarrow$ node $6 \rightarrow$ node 8 (38)

Path $2_{b}$ : node $1 \rightarrow$ node $4 \rightarrow$ node $6 \rightarrow$ node $7 \rightarrow$ node 8 (40)

Path $3_{b}$ : node $1 \rightarrow$ node $4 \rightarrow$ node $6 \rightarrow$ node $5 \rightarrow$ node 8 (44)

Path $4_{b}$ : node $1 \rightarrow$ node $4 \rightarrow$ node $2 \rightarrow$ node $6 \rightarrow$ node 8 (38)

Path $5_{\mathrm{b}}$ : node $1 \rightarrow$ node $4 \rightarrow$ node $2 \rightarrow$ node $5 \rightarrow$ node 8 (44)

Path $6_{b}$ : node $1 \rightarrow$ node $4 \rightarrow$ node $2 \rightarrow$ node $6 \rightarrow$ node $5 \rightarrow$ node 8 (44)

Path 7 : node $1 \rightarrow$ node $4 \rightarrow$ node $2 \rightarrow$ node $6 \rightarrow$ node $7 \rightarrow$ node 8 (40)

There are more paths from node 1 to node 8 which follow the path from node 1 to node 4 and node 2 to node 8 . The paths from node 2 to node 8 are specified above.

In the cases of both transmissions, all the possible paths and the bandwidth available for each path are determined. Then calculation of the levels of congestion of all the determined paths is done and the same data is communicated to the transport layer utilizing the cross layer technique.

The nodes disjoint test is to be carried out now. After this test, it is determined that path $1_{b}$ is a node disjoint with path $4_{a}$, path $2_{b}$ is a node disjoint with path $4_{b}$. In the same way, the nodes disjoint test is carried out in all the possibilities. Finally, path $4_{\mathrm{a}}$ is selected for the first transmission (node 2 -> node 8 ) and path $2 \mathrm{~b}$ or path $7 \mathrm{~b}$ is selected for the second transmission (node 1 -> node 8 ). As the nodes disjoint test is successful in the example considered, there is no further need to go for edge any disjoint test or further process.

\section{Performance evaluation}

In this section, the obtained results of the proposed work, which is simulated using Network Simulator NS-2, are presented. The performance evaluation is done on the basis of the parameters, such as size of the sending window, number of dropped packets, number of received packets and the delay ratio. The parameters are defined below: 
- Sending the window size: The maximum number of bytes that can be transmitted at any point of time is referred as sending the window size.

- Number of packets dropped: The number of packets that are received by the receiver or reached the receiver, transmitted by the sender.

- Number of packets received: The number of packets received by the receiver, transmitted by the sender.

- Delay: The time elapse by which the receiver receives the packets since the time of the sender transmission of the packets.

Results and discussions. The obtained results for analysis are collected from the average values taken from among 30 runs of the simulation which is carried out in $140 \mathrm{~s}$. From the results obtained by the trace graph of the simulator, the proposed algorithm, CLCC, which is a congestion control procedure in the wireless network based on the cross layer technique, is observed to have better control over the bandwidth management issues in the network and enhances the network performance in terms of ratio of delay, dropped packets, received packets and size of the sending window. The data transmission time and speed with a constant bit rate and the packet transmission capability are used to evaluate the transmitted packets, received packets and dropped packets. The proposed algorithm, CLCC performs better in comparison with WCPCap. Table 1 displays the parameters used in the process of the simulation process.

Table 1. Simulation parameters

\begin{tabular}{|l|l|}
\hline TCP & newReno \\
\hline Initial Sending Window Size & 150 \\
\hline Sender Rate & $10 \mathrm{Mb}$ \\
\hline Sender side delay & $5 \mathrm{~ms}$ \\
\hline Receiver Rate & $2 \mathrm{Mb}$ \\
\hline Receiver side delay & $40 \mathrm{~ms}$ \\
\hline Queue limit & 10 \\
\hline Packet Size & $750 \mathrm{~b}$ \\
\hline Routing protocols & AODV \\
\hline
\end{tabular}

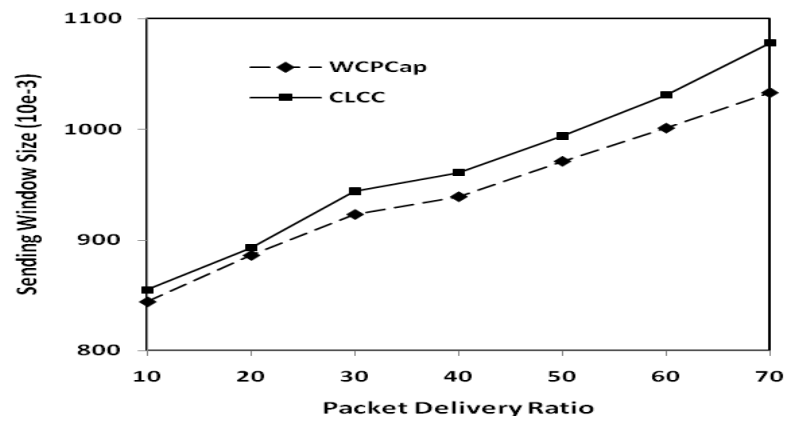

Fig. 5. Comparison of WCPCap and CLCC in terms of the sending window size

In Fig. 5 the size of the sending window is provided for the purpose of comparison among various mechanisms for window control, such as congestion 
control mechanism based on the cross layer for wireless mesh networks and Wireless Control Protocol along with capacity Estimation Protocol (WCPCap). The unit of the size of congestion window is Mb. The sending window size determines the maximum quantity or the number of packets which can be transmitted. In case of CLCC the decrement in the congestion window size is comparatively less since the reduction is only $25 \%$ in the event of a transmission failure. In comparison to WCPCap, the congestion window is always maintained high as it can be observed. If the size of the congestion is larger, a larger number of packets can be transmitted. As CLCC mechanism can transmit a bigger number of packets, numerous applications like long mile Internet and high speed video applications can be efficiently managed.

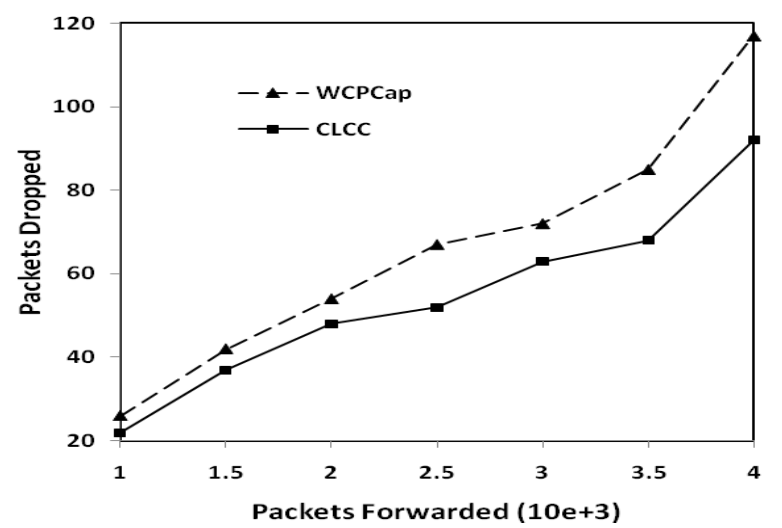

Fig. 6. Comparision of WCPCap and CLCC in terms of the number of dropped packets

In Fig. 6 a comparison of the number of dropped packets against the number of forwarded packets is provided for various mechanisms of window control, such as the congestion control based on the cross layer wireless mesh networks and Wireless Control Protocol with Capacity Estimation protocol (WCPCap). The proposed algorithm CLCC is observed to drop a smaller number of packets in comparison to WCPCap, due to the process of selection of the path for transmitting the packets. Similarly, in Fig. 7, a comparison of the number of received packets against the number of forwarded packets is provided for various mechanisms of the window control, such as the congestion control based on the cross layer wireless mesh networks and Wireless Control Protocol with Capacity Estimation protocol (WCPCap). The packet drops illustrated in Fig. 5 imply this result. In proportion to the reduction in the number of dropped packets, it is observed that there is an obvious and corresponding increase in the number of received packets by the receiver. The proposed algorithm CLCC to receives a larger number of packets with respect to the smaller number of packets in WCPCap. This becomes possible because of the path selection process for packets transmission and prior estimation of the possible congestion. 


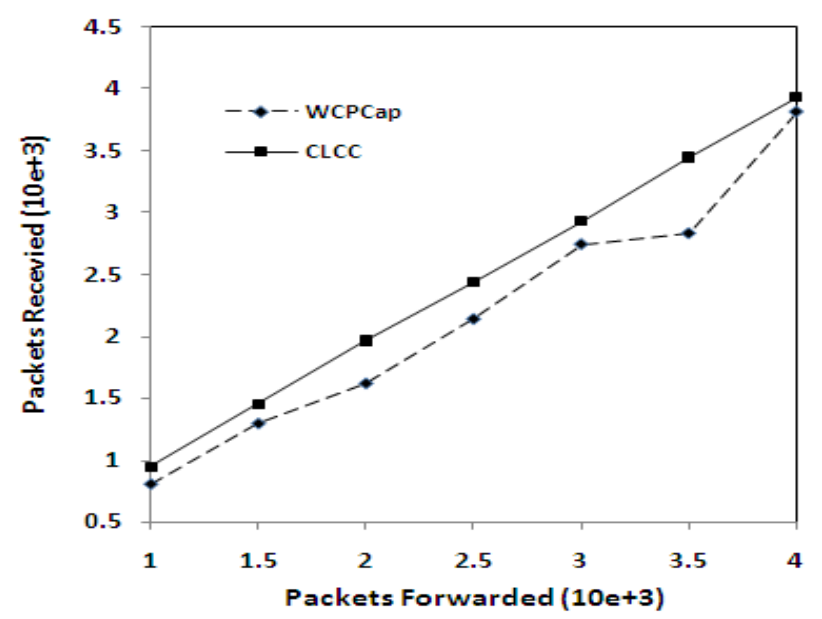

Fig. 7. Comparision of WCPCap and CLCC in terms of the number of packets received

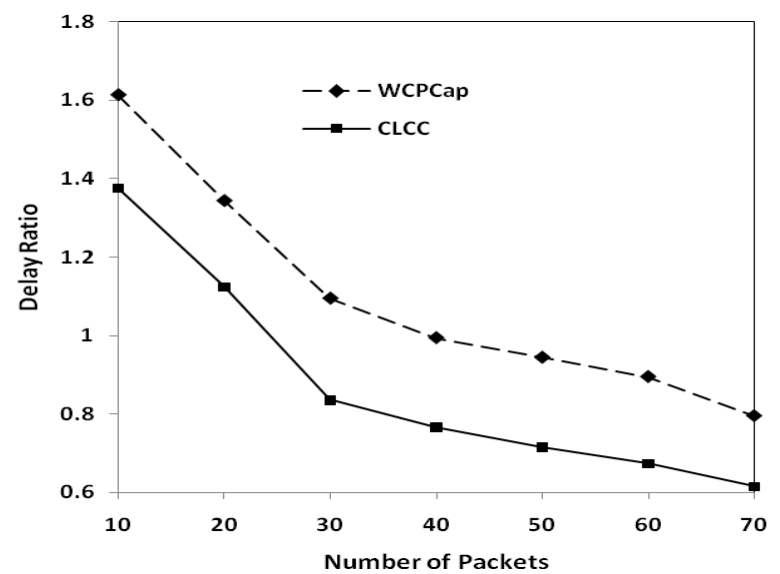

Fig. 8. Comparision of WCPCap and CLCC in terms of the delay ratio

Fig. 8 demonstrates the delay performance of the proposed algorithm CLCC and WCPCap. The delay ratio is obtained through estimation of the packets delivery between the source and the destination. The delay in WCPCap is observed to be high when compared to CLCC, as displayed in Fig. 8. The reason for this is the fact that the path is selected on the basis of the available and required bandwidth.

\section{Conclusions and future work}

In this paper we have proposed an effective congestion control mechanism called CLCC. The bandwidth utilization in the proposed algorithm, CLCC, is efficient when compared to other strategies. Consequently it is evident that the congestion window size is much larger in CLCC than in the others. The proposed model with efficient utilization of the bandwidth through congestion control attracts many applications, such as campus networking, high speed Internet applications, public 
safety and transport systems. We have plans for extending this framework further for inclusion of media protocols, which can consider the cross layer interaction, so that QoS assurances for real time applications can be provided. We also intend to develop algorithms capable of giving efficient access to channels even during the high load phases and instances on the network, negotiating the issue of congestion and maintaining stability in the network.

\section{References}

1. Akyildiz, I. F., X. Wang, W. Wang. Wireless Mesh Networks: A Survey. - Computer Networks, Vol. 47, March 2005, 445-487.

2. We i, Y., R. Ma o sheng, T. Zha o, L. Xia o ming. A Bandwidth Management Scheme Support for Real-Time Applications in Wireless Mesh Networks. - In: Proceedings of the 2008 ACM Symp. on Applied Computing (SAC’08), 2063-2068, doi: $10.1145 / 1363686.1364185$.

3. Y a n g, D e-N i a n, M i n g-S y a n C h e n. Efficient Resource Allocation for Wireless Multicast. IEEE Transactions on Mobile Computing, Vol. 7, April 2008, No 4, 387-400.

4. N a hr s t e d t, K., S. S hah, K. C he n. Cross-Layer Architectures for Bandwidth Management in Wireless Networks. - Resource Management in Wireless Networking, Springer, Vol. 16, 2005, Section I, 41-62.

5. R a n g w a la, S., A. J in d a l, K i-Y o ung J an g, K. P s o u n i s, R. G o vind a n. NeighborhoodCentric Congestion Control for Multihop Wireless Mesh Networks. - Networking, IEEE/ACM Transactions on, Vol. 19, December 2011, No 6, 1797-1810. doi: 10.1109/TNET.2011.2146272.

6. X u, S., T. S a a d a w i. Performance Evaluation of TCP Algorithms in Multi-Hop Wireless Packet Networks. - Journal of Wireless Communications and Mobile Computing, Vol. 2, 2002, No $1,85-100$.

7. I q b a l, M., X. W a n g, S. L i, T. E 11 i s. QoS Scheme for Multimedia Multicast Communications Over Wireless Mesh Networks. - IET Commun.,Vol. 4, 2010, Issue 11, 1312-1324.

8. Venkat a Kri shna, P., V. S a r i tha, G. Vedha, A. B hiwa l, A. S. Chaw la. Quality-OfService-Enabled Ant Colony-Based Multipathrouting for Mobile Ad-Hoc Networks. - IET Commun., Vol. 6, 2012, Issue 1, 76-83.

9. Ven kata Krishna, P., I y e n gar. A Cross Layer Based Qos Model for Wireless and Mobile Networks. - Journal of Mobile Communication, Vol. 1, 2007, No 4, 114-120.

10. Venkata Krishna, P. A Study of Quality of Service Metrics for Wireless Systems. Ph. D Thesis, VIT University, 2008.

11. Misra, S., P. Venkata Krishna, A. Bhiwal, A. S. Chawla, B. E. Wolfinger, C. L e e. A Learning Automata-Based Fault-Tolerant Routing Algorithm for Mobile Ad Hoc Networks. - The Journal of Supercomputing, Springer Netherlands. doi: 10.1007/s11227011-0639-8.

12. Misra, S., P. Venkata Krishna, V. Saritha. LACAV: An Energy-Efficient Channel Assignment Mechanism for Vehicular Ad-Hoc Networks. - The Journal of Supercomputing, Springer Netherlands. doi: 10.1007/s11227-011-0552-1.

13. Misra, S., P. Venkata Krishna, V. Saritha. An Efficient Approach for Distributed Channel Allocation with Learning Automata-Based Reservation in Cellular Networks. SIMULATION , 2011. doi: 10.1177/0037549711420175.

14. M i sra, S., P. V e n k a t a Kri is hna, K. I. A bra ha m. Learning Automata Solution for Medium Access with Channel Reservation in Wireless Networks. - Wireless Personal Communications (WPS), Springer, Vol. 56, 2011, Issue 1, 55-72.

15. Ve n k a t a K ri shn a, P., S. M i s r a, M. S. O b a i d a t, V. S a rit h a. Virtual Backoff Algorithm: An Enhancement to 802.11 Medium Access Control to Improve the Performance of Wireless Networks. - IEEE Trans. on Vehicular Technology (VTS), Vol. 59, 2010, No 3, 1068-1075.

16. Venkata Krishna, P., S. Misra, M. S. Obaidat, V. Saritha. An Efficient Approach for Distributed Dynamic Channel Allocation with Queues for Real Time and Non Realtime Traffic in 
Cellular Networks. - Elsevier, Journal of Systems \& Software, Vol. 82, 2009, 1112-1124. doi: 10.1016/j.jss.2009.01.043.

17. V e n k a t a K ri s h n a, P., N. C h. S. N. I y e n g a r. Design of Sequencing Medium Access Control to Improve the Performance of Wireless Networks. - Journal of Computing and Information Technology (CIT Journal), Vol. 16, June 2008, No 2, 81-89.

18. Venkata Krishna, P., N. Ch. S. N. I y engar, S. Misra. An Efficient Hash Table Node Identification Method for Bandwidth Reservation in Hybrid Cellular and Ad-Hock Networks. - Computer Communications, Elsevier, Vol. 31, March 2008, No 4,722-733.

19. V e n k a t a Kri shn a, P., N. Ch. S. N. I y e n gar. Optimal Channel Allocation Algorithm with Efficient Channel Reservation for Cellular Networks. - Int. J. Communication Networks and Distributed Systems, Vol. 1, 2008, No 1, 33-51.

20. V e n k a t a K ri sh na, P., N. C h. S. N. I y e n g a r. Sequencing Technique - An Enhancement to 802.11 Medium Access Control to Improve the Performance of Wireless Networks. - Int. J. Communication Networks and Distributed Systems, Vol. 1, 2008, No 1, 52-70.

21. Saha, M., P. Venkata Krishna. Bandwidth Management Framework for Multicasting in Wireless Mesh Networks. - In: 2011 International Conference on Network Communication and Computer (ICNCC'2011), 295-299.

22. Venkata Krishna, P., S. Misra, M. S. Obaidat, V. Saritha. A New Scheme for Distributed Channel Allocation in Cellular Wireless Networks. - In: Proc. of 12th ACM/SCS Communications and Networking Simulation Symposium (CNS), San Deigo, USA, March 2009, 22-27.

23. Venkata Krishna, P., S. Misra, M. S. Obaidat, V. Saritha. An Efficient 802.11 Medium Access Control Method and Its Simulation Analysis. - In: Proc. of 7th ACS/IEEE International Conference on Computer Systems and Applications, AICCSA 2009, Rabat, Morocco, 10-13 May 2009, 330-335 (accepted for publication).

24. Venkata Krishna, P., N. Ch. S. N. I yengar, S. Misra. An Approach for Bandwidth Reservation in Ad-Hoc Networks Having Infrastructure Support. - In: Proc. of. 7th WSEAS International Conference on Applied Computer Science (ACS’2007), Italy, November 2007.

25. Ganesh, A. J., D. Polis, P. B. Key, R. Srikant. Congestion Notification and Probing Mechanisms for Endpoint Admission Control. - IEEE/ACM Transactions on Networking, Vol. 14, June 2006, No 3, 568-578.

26. B y u n, H e e-J u n g, J o n g-T a e L i m. On a Fair Congestion Control Scheme for TCP Vegas. IEEE Communications Letters, Vol. 9, February 2005, No 2, 190-192.

27. W a n g, L., L. W a n g, S. J i a. A Flow Control Scheme for Wireless ATM with Hybrid ARQ and Weighted ERICA Algorithm Combined. - In: Proc. of 14th Int. Symposium on Personal, Indoor and Mobile Radio Communication, 2003, 2538-2542.

28. Kunni yur, S., S. Kunni yur. End-to-End Congestion Control Schemes: Utility Functions, Random Losses and ECN Marks. - IEEE/ACM Transactions on Networking, Vol. 11, October 2003, No 5, 689-702.

29. Flo y d, S., V. J a c o b s o n. Random Early Detection Gateways for Congestion Avoidance. IEEE/ACM Transactions on Networking, August 1993.

30. H o u, T.-C., C.-W. H s u, C.-S. W u. A Delay-Based Transport Layer Mechanism for Fair TCP Throughput over 802.11 Multihop Wireless Mesh Networks. - Int. J. Commun. Syst., Vol. 24, 2011, 1015-1032. doi: 10.1002/dac.1207.

31. L i n, P. J., C. R. Dow, P. H suan, S. F. Hwang. An Efficient Traffic Control System Using Dynamic Thresholding Techniques in Wireless Mesh Networks. - Int. J. Commun. Syst., Vol. 24, 2011, 325-346. doi: 10.1002/dac.1157.

32. V a l a r m a t h i, K., N. M a l m u r u g a n. Distributed Multichannel Assignment with Congestion Control in Wireless Mesh Networks. - Int. J. Commun. Syst., Vol. 24, 2011, 1584-1594. doi: 10.1002/dac.1234.

33. L e e, C., T. J e on g, S. L i a n. Tournament-Based Congestion Control Protocol for Multimedia Streaming In Ubiquitous Sensor Networks. - Int. J. Commun. Syst., Vol. 24, 2011, 1246-1260. doi: 10.1002/dac.1179.

34. K o, E., D. A n, I. Y e o m, H. Y o o n. Congestion Control for Sudden Bandwidth Changes in TCP. - Int. J. Commun. Syst., Vol. 25, 2012, 1550-1567. doi: 10.1002/dac.1322. 
35. Ulud a g, S., T. I m b o d e n, K. A k k a y a. A Taxonomy and Evaluation for Developing 802.11Based Wireless Mesh Network Testbeds. - Int. J. Commun. Syst., Vol. 25, 2012, 963-990. doi: 10.1002/dac.1299.

36. Ci c c on e t t i, C., I. F. A k y il di z, L. L e n z in i. FEBA: A Bandwidth Allocation Algorithm for Service Differentiation in IEEE 802.16 Wireless Mesh Networks. - IEEE/ACM Trans. on Networking, Vol. 17, June 2009, No 3, 884-897.

37. B a k h s h i, B., S. K h or s a n di. On the Performance and Fairness of Dynamic Channel Allocation in Wireless Mesh Networks. - International Journal of Communication Systems, Vol. 26, 2013, No 3, 293-314.

38. Misra, S., T. I. G ho sh, M. S. Oba idat. Routing Bandwidth Guaranteed Paths for Traffic Engineering in WiMAX Mesh Networks. - International Journal of Communication Systems, 2013 (in Press).

39. Y a ng, S.-F., J.-S. Wu, B.-J. Hwang. Performance Evaluation of Priority Based Adaptive Multiguard Channel Call Admission Control for Multiclass Services in Mobile Networks. International Journal of Communication Systems, Vol. 26, 2013. No 5, 597-609. 